\section{Atendimentos decorrentes de queimaduras em serviços públicos de emergência no Brasil, 2009}

\author{
Public hospital emergency department visits due \\ to burns in Brazil, 2009
}

Vilma Pinheiro Gawryszewski 1 Regina Tomie Ivata Bernal 2 Nilza Nunes da Silva 2 Otaliba Libânio de Morais Neto ${ }^{3}$ Marta Maria Alves da Silva ${ }^{3}$ Márcio Dênis Medeiros Mascarenhas 4 Naíza Nayla Bandeira de Sá 3 Rosane Aparecida Monteiro 5 Deborah Carvalho Malta ${ }^{3}$

\title{
Introdução
}

1 Pan American Health Organization, World Health Organization, Washington DC, U.S.A.

2 Faculdade de Saúde Pública, Universidade de São Paulo, São Paulo, Brasil. 3 Secretaria de Vigilância em Saúde, Ministério da Saúde, Brasília, Brasil. ${ }^{4}$ Universidade Federal do Piauí, Teresina, Brasil.

5 Faculdade de Medicina de Ribeirão Preto, Universidade de São Paulo, Ribeirão Preto, Brasil.

Correspondência V. P. Gawryszewski Pan American Health Organization, World Health Organization. 525 23rd St NW, Washington DC 20037-2895, U.S.A. gawry@uol.com.br

\section{Abstract}

The objective was to analyze the characteristics of burn injuries treated in emergency departments (ED) and associated factors. This was a crosssectional study of 761 ED visits collected through the National Injury Surveillance System in 2009. The majority of patients were males (58.6\%), and the most prevalent age brackets were 30-49 years (23.1\%) and 0-4 years (23\%). Most burns occurred at home (62.1\%), especially among females and children, and in commerce/services/industry/ construction (19.1\%), mainly among males 20-49 years. Work-related burns comprised $29.1 \%$ of the overall sample. Alcohol use prior to the injury was reported in $5.1 \%$ of cases. Causal agents across all age brackets were: contact with hot substances (43.6\%) and exposure to fire and flames (24.2\%); among the economically productive age groups, association with chemicals substances was common. Burns in children 0-14 years were associated with injuries at home, contact with heat and hot substances, and subsequent hospitalization; burns in the 15-49-year bracket were associated with exposure to firelflames and electrical current, injuries occurring in public places, and outpatient treatment and discharge. The study highlights the importance of burn prevention strategies targeting children and workers.

Burns; Emergency Medical Services; External Causes
As queimaduras determinam intensa dor física, que envolve não somente o acidentado, mas também seus familiares e círculo de relações. Estudo conduzido em Unidade de Queimados de hospital em Ribeirão Preto, São Paulo, Brasil, considerou que a dor do paciente queimado é "terrível para quem sente, estressante para quem cuida", apontando o sofrimento vivido também por cuidadores, especialmente na hora do banho e curativos, considerados os momentos mais dolorosos para esses pacientes 1 . Além disso, dependendo da extensão e gravidade, podem causar sequelas físicas e psíquicas que podem diminuir as chances dos indivíduos afetados de usufruir plenamente de seu potencial produtivo do ponto de vista econômico e social. O impacto que as deformidades causam na vida dessas pessoas é difícil de ser medido.

O estudo de Mock et al. 2 estima que mais de 300 mil pessoas morrem de queimaduras por fogo sem incluir as outras mortes decorrentes de queimaduras químicas, contato com substâncias quentes, eletricidade, entre outras. Além disso, chama atenção que cerca de $95 \%$ dessas mortes ocorrem nos países de baixa e média rendas, exemplificando que as taxas de mortalidade variam de 11,6/100 mil, média dos países do Sudeste da Ásia, até 1,0/100 mil, média dos países desenvolvidos. No Brasil, apesar de não determinarem um forte impacto no perfil da mortalidade 
da população, têm alta relevância na morbidade. Isso pode ser mais bem evidenciado a partir da análise das fontes oficiais de informação de saúde. Os dados preliminares referentes ao ano de 2009, disponibilizados pelo Sistema de Informações sobre Mortalidade - SIM (Departamento de Informática do SUS; http://www.datasus.gov. br), mostraram que o número de vítimas fatais por essa causa foi 2.175, representando cerca de $1,6 \%$ do total de mortes decorrentes de causas externas. Já em relação às vítimas não fatais, a tabulação dos dados do Sistema de Informações Hospitalares do Sistema Único de Saúde (SIH/ SUS; http://www.datasus.gov.br) para o ano de 2009 mostrou que 80.607 pessoas foram internadas no sistema público de saúde brasileiro por lesões decorrentes de queimaduras nesse ano, representando cerca de $9 \%$ do total de internações do grupo das causas externas.

Especialmente nos países classificados como de média e baixa rendas, as queimaduras ainda permanecem como um dos agravos mais negligenciados entre os vários tipos de causas externas. Já nos países desenvolvidos, a redução nas taxas de mortalidade e lesões por queimaduras vem sendo alcançada como resultado de uma série de intervenções, tais como a promoção do uso de detectores de fumaça em residências e edifícios, a instalação de sistemas que jorram água nos tetos, o aumento da segurança nos dispositivos de combustíveis domésticos, a diminuição da temperatura dos aquecedores de água quente, entre outros ${ }^{2}$. Além disso, também contribuíram para tal redução todos os avanços no tratamento e cuidado de pacientes queimados e da queimadura obtidos nas últimas décadas.

Por essas razões, torna-se urgente dar maior visibilidade ao problema e, ao mesmo tempo, fornecer os subsídios científicos necessários para orientar as ações de prevenção. Nesse aspecto, considera-se que a análise dos dados provenientes do Sistema Nacional de Serviços Sentinelas de Vigilância de Violências e Acidentes (VIVA), que é um inquérito acerca dos atendimentos decorrentes de causas externas realizados em serviços de urgência e emergência em todo o Brasil, e que foi implantado pelo Ministério da Saúde em 2006, pode contribuir grandemente para aumentar a compreensão desse agravo ${ }^{3}$. Dessa maneira, o presente estudo tem como objetivo descrever as características dos atendimentos decorrentes de queimaduras em serviços de urgência e emergência selecionados, e analisar possíveis fatores associados com base nos dados coletados pelo VIVA em 2009.

\section{Metodologia}

Os dados do presente trabalho referem-se ao total de 761 atendimentos decorrentes de queimaduras para o período de 30 dias, extraídos do banco do VIVA, inquérito 2009, cuja metodologia encontra-se detalhada em outras publicações 4. Seguindo a 10a revisão da Classificação Internacional de Doenças (CID-10), entre essas queimaduras foram incluídos os casos decorrentes de exposição à corrente elétrica, radiação e temperatura; exposição à fumaça, fogo e chamas; e o contato com fonte de calor e substâncias quentes. O projeto de pesquisa foi aprovado pela Comissão Nacional de Ética em Pesquisa (CONEP). Por se tratar de ação de vigilância epidemiológica de âmbito nacional, a assinatura do Termo de Consentimento Livre e Esclarecido foi substituída por consentimento verbal, obtido pelo paciente ou por seu responsável, registrado em campo da ficha de coleta.

\section{Delineamento e população do estudo}

Trata-se de um estudo transversal, cujos dados foram coletados no período de 30 dias consecutivos entre setembro e novembro de 2009, em turnos de 12 horas selecionados mediante sorteio probabilístico em serviços habilitados para o atendimento de urgência e emergência no âmbito do Sistema Único de Saúde (SUS). A população do estudo foi composta pelas vítimas de causas externas (acidentes e violências) que procuraram atendimento nos serviços de urgência e emergência dos municípios selecionados. Como referido anteriormente, o presente trabalho está focalizado no subgrupo composto pelas vítimas de queimaduras.

As entrevistas foram realizadas por acadêmicos e profissionais de saúde, sob a supervisão de técnicos das secretarias de saúde. A padronização dos procedimentos de coleta foi assegurada por meio de treinamentos para os técnicos das secretarias estaduais e municipais de saúde, ministrados pela equipe da Coordenação de Vigilância de Doenças e Agravos Não Transmissíveis da Secretaria de Vigilância em Saúde, Ministério da Saúde. Os dados foram digitados pelos municípios no programa Epi Info 3.5.1 (Centers for Disease Control and Prevention, Atlanta, Estados Unidos). A análise de consistência e duplicidades foi realizada utilizando-se o programa Link Plus, versão 2.0 (CDC’s Division of Cancer Prevention and Control, Atlanta, Estados Unidos). 


\section{Local do estudo}

A pesquisa incluiu 74 serviços de urgência e emergência situados no Distrito Federal e em 23 capitais de estados. Os serviços foram selecionados segundo os seguintes critérios: ser referência para atendimento às causas externas na área de urgência e emergência, número de atendimentos realizados, complexidade e resolutividade do serviço. Além disso, foi realizada consulta ao $\mathrm{Ca}$ dastro Nacional de Estabelecimentos de Saúde (CNES) e aos registros do SIH/SUS.

Tamanho da amostra e processo de amostragem

Para a definição do tamanho da amostra, considerou-se o critério de precisão para as estimativas de prevalências fixadas para o estudo (coeficiente de variação inferior a 30\% e o erro-padrão menor que 3). Desse modo, o tamanho mínimo da amostra foi de 1.500 atendimentos por causas externas nas capitais de estados.

Trata-se de uma amostra por conglomerado 5 em único estágio de seleção, na qual a unidade primária de amostragem foi o turno de 12 horas. Para efeito de sorteio de turnos considerou-se o período de coleta de 30 dias dividido em dois turnos, totalizando 60 turnos, sendo 30 diurnos (07:00 às 18:59) e 30 noturnos (19:00 às 06:59). $O$ número de turnos a ser sorteado em cada estabelecimento foi obtido pela razão entre o tamanho mínimo da amostra de atendimentos (2009) por causas externas e a média de atendimentos por essas causas realizada no mesmo estabelecimento em anos anteriores. Para garantir o tamanho mínimo da amostra, nas capitais, foram sorteados dois turnos reservas.

\section{Análises estatísticas}

Inicialmente, foi realizado o estudo descritivo dos casos decorrentes de queimaduras. Para verificar possíveis associações entre as variáveis levantadas foi utilizada a técnica de análise de correspondência, uma vez que essa técnica permite trabalhar com uma grande quantidade de variáveis qualitativas, constituídas de grande número de categorias 6,7 . Essa análise é equivalente à análise fatorial, porém os resultados são apresentados de forma gráfica, na qual as menores distâncias entre as categorias linha e categorias coluna representam as mais fortes associações entre elas, enquanto que as maiores distâncias representam dissociações entre as mesmas ${ }^{8}$. Foram calculadas as tabelas de contingência expandidas (total de atendimentos) e com base nelas foi obtido o gráfico de correspondência. $\mathrm{O}$ estimador ${ }^{9}$ do to- tal de atendimentos por acidentes e violências em serviços sentinelas de urgência e emergência no período de 30 dias é dado pela expressão:

$$
\hat{Y}=\sum_{h=1}^{L} \sum_{i=1}^{n_{h}} \sum_{j=1}^{m_{h i}} w_{h i j} y_{h i j}
$$

Em que, $w_{\text {hij }}$ : peso da amostra no h-ésimo estrato (CNES), i-ésima UPA (turno) e j-ésimo número de elementos do h-ésimo estrato da íésima UPA; $\mathrm{y}_{\mathrm{hij}}$ : valor observado da variável (1 se possui e 0 em caso contrário) no h-ésimo estrato, i-ésima UPA e j-ésimo número de elementos do h-ésimo estrato da í-ésima UPA.

\section{Resultados}

\section{Perfil das vítimas de queimaduras}

Para caracterizar o perfil dos 761 atendimentos decorrentes de queimaduras na amostra do conjunto das 23 capitais e do Distrito Federal, no período de setembro a novembro de 2009, foram selecionadas as variáveis apresentadas nas Tabelas 1 e 2. Deve ser assinalado que o total de atendimentos estimado para o mês pesquisado alcança 2.540. A Tabela 1 mostra o predomínio dos homens (446 casos; $58,6 \%$ do total) em relação às mulheres nesses atendimentos (315 casos; $41,4 \%$ do total). As faixas etárias mais acometidas foram crianças, adolescentes e os indivíduos adultos em idade produtiva, uma vez que a faixa dos 20 a 29 anos (23,1\%) ocupou o primeiro lugar, seguindo-se as crianças menores de 10 anos (22\%). Entre as crianças, chamou atenção a faixa etária de 0 a 4 anos por representar $16 \%$ do total de atendimentos. As pessoas idosas com 50 anos ou mais de idade foram as com menor representação percentual entre os atendidos por queimaduras. Essas mesmas faixas etárias foram as mais acometidas no sexo masculino, porém, no sexo feminino os indivíduos com idades entre 0 e 9 anos e 20 a 29 apresentaram proporções similares. Quanto à raça/cor da pele, os de raça/ cor parda representaram $43 \%$ do total de atendimentos, seguindo-se os brancos $(35,9 \%)$ e os pretos (13,5\%). Comparativamente aos homens, as mulheres apresentaram um percentual maior de pessoas de cor parda $(39,2 \%$ e $48,2 \%$, respectivamente). Quanto à escolaridade, a maioria dos pacientes atendidos referiu ter entre 9 e 11 anos de estudos $(27,1 \%)$, seguida daqueles que referiram ter entre 0 e 4 anos (25,2\%). As menores proporções foram encontradas entre aqueles com 12 anos e mais de estudos. Foram observadas algumas diferenças na escolaridade dos homens e mulheres atendidos por queimaduras, uma vez que os primeiros apresentaram-se 
Atendimentos decorrentes de queimaduras segundo características demográficas: proporção de casos (\%), coeficiente de variação (CV). Serviços de urgência e emergência selecionados em 23 capitais e no Distrito Federal, Brasil, setembro a novembro de 2009.

\begin{tabular}{|c|c|c|c|c|c|c|}
\hline \multirow[t]{2}{*}{ Características } & \multicolumn{2}{|c|}{ Masculino $(n=446)$} & \multicolumn{2}{|c|}{ Feminino $(n=315)$} & \multicolumn{2}{|c|}{ Total $(\mathrm{N}=761)$} \\
\hline & $\%$ & CV (\%) & $\%$ & CV (\%) & $\%$ & CV (\%) \\
\hline \multicolumn{7}{|l|}{ Faixa etária (anos) } \\
\hline $0-4$ & 17,0 & 12,8 & 14,7 & 14,3 & 16,0 & 9,4 \\
\hline $5-14$ & 9,8 & 18,2 & 13,7 & 16,0 & 11,5 & 12,8 \\
\hline $15-19$ & 9,5 & 16,1 & 9,6 & 19,3 & 9,6 & 12,5 \\
\hline $20-29$ & 23,8 & 11,9 & 22,1 & 11,5 & 23,1 & 8,3 \\
\hline $30-49$ & 29,0 & 8,6 & 27,5 & 9,9 & 28,4 & 6,6 \\
\hline 50 e + & 10,5 & 16,4 & 12,3 & 21,5 & 11,3 & 14,2 \\
\hline Sem informação & 0,5 & - & - & - & 0,3 & \\
\hline \multicolumn{7}{|l|}{ Raça/Cor } \\
\hline Branca & 36,9 & 10,3 & 34,6 & 9,9 & 35,9 & 8,0 \\
\hline Preta & 14,0 & 16,1 & 12,8 & 15,8 & 13,5 & 13,3 \\
\hline Parda & 39,2 & 9,4 & 48,2 & 7,5 & 43,0 & 6,8 \\
\hline Amarela/Indígena & 3,1 & 32,7 & 1,7 & 40,5 & 2,5 & 27,1 \\
\hline Sem informação & 6,8 & - & 2,8 & - & 5,1 & \\
\hline \multicolumn{7}{|l|}{ Escolaridade (anos) } \\
\hline $0-4$ & 23,8 & 11,8 & 27,2 & 9,5 & 25,2 & 7,6 \\
\hline $5-8$ & 24,1 & 11,3 & 18,6 & 14,0 & 21,8 & 8,7 \\
\hline $9-11$ & 24,1 & 11,9 & 31,2 & 9,3 & 27,1 & 7,7 \\
\hline 12 e + & 5,0 & 23,9 & 5,1 & 26,1 & 5,1 & 17,7 \\
\hline Não se aplica * & 14,3 & - & 12,2 & - & 13,4 & - \\
\hline Sem informação & 8,8 & - & 5,8 & - & 7,5 & - \\
\hline
\end{tabular}

* Casos de queimaduras em crianças menores de sete anos.

homogeneamente distribuídos entre 0 e 11 anos de estudos, enquanto as mulheres apresentaram proporções maiores para escolaridade: de 9 a 11 anos e 0 a 4 anos.

A Tabela 2 mostra que um pouco mais da metade dessas vítimas utilizou o veículo particular (54\%) como meio de locomoção até o serviço de emergência, seguindo-se aqueles que chegaram a pé ou utilizaram transporte coletivo $(20,9 \%)$ e os que foram atendidos por unidades do Serviço de Atendimento Móvel de Urgência (SAMU), ambulância e resgate (19,8\%). A presença de algum tipo de deficiência (física, mental, visual, auditiva, outras deficiências/síndromes) foi relatada por uma pequena proporção de casos: somente $2,2 \%$ de todos os atendimentos. Por outro lado, em $29,1 \%$ dos atendimentos foi referido que o evento esteve relacionado ao trabalho, sendo que esta proporção foi maior no sexo masculino $(35,1 \%)$ comparativamente ao sexo feminino (20,8\%). O consumo de álcool declarado pela vítima ou suspeito pelo entrevistador chegou a
$5,1 \%$, sendo que o percentual encontrado entre os homens é cerca de o dobro do das mulheres (6,5\% e $3 \%$, respectivamente).

As queimaduras ocorreram predominantemente nas residências $(62,1 \%$ do total de atendimentos). O local de ocorrência que ocupou o segundo lugar foi o comércio/serviços/indústria/ construção, onde aconteceram 19,1\% dos atendimentos. Seguiram-se a via pública e habitação coletiva com percentuais de 7,6\% e 3,8\%, respectivamente. A distribuição do local de ocorrência segundo sexo mostrou diferenças, uma vez que as mulheres sofreram queimaduras nas residências em proporções mais altas que os homens $(77,6 \%$ e 50,8\%, respectivamente). Em posição inversa encontrou-se o comércio, serviços, indústria e construção, onde $26 \%$ dos homens sofreram queimaduras enquanto que este foi o local de ocorrência para somente $6,3 \%$ das mulheres.

Em relação à natureza da lesão, as queimaduras foram $95 \%$ dos casos, sendo que os $4,9 \%$ restantes foram diagnosticados como contusões, 
Atendimentos decorrentes de queimaduras segundo circunstâncias das ocorrências: proporção de casos (\%), coeficiente de variação (CV). Serviços de urgência e emergência selecionados em 23 capitais e no Distrito Federal, Brasil, setembro a novembro de 2009.

\begin{tabular}{|c|c|c|c|c|c|c|}
\hline \multirow[t]{2}{*}{ Características } & \multicolumn{2}{|c|}{ Masculino $(n=446)$} & \multicolumn{2}{|c|}{ Feminino $(n=315)$} & \multicolumn{2}{|c|}{ Total $(\mathrm{N}=761)$} \\
\hline & $\%$ & CV (\%) & $\%$ & CV (\%) & $\%$ & CV (\%) \\
\hline \multicolumn{7}{|c|}{ Meio de transporte utilizado para chegar ao hospital } \\
\hline A pé/Coletivo & 20,1 & 13,8 & 21,8 & 12,6 & 20,9 & 10,4 \\
\hline Veículo particular & 53,2 & 7,9 & 55,2 & 6,1 & 54,0 & 5,5 \\
\hline Assistência pré-hospitalar * & 22,8 & 20,7 & 15,7 & 13,0 & 19,8 & 14,8 \\
\hline Outros & 2,8 & 36,9 & 2,8 & 32,8 & 2,8 & 28,6 \\
\hline Sem informação & 1,1 & - & 4,5 & - & 2,5 & - \\
\hline \multicolumn{7}{|l|}{ Possui algum tipo de deficiência } \\
\hline Sim & 2,8 & 33,5 & 2,3 & 39,6 & 2,6 & 28,0 \\
\hline \multicolumn{7}{|l|}{ Evento relacionado ao trabalho } \\
\hline Sim & 35,1 & 10,4 & 20,8 & 14,0 & 29,1 & 8,8 \\
\hline \multicolumn{7}{|l|}{ Uso de álcool ** } \\
\hline Sim & 6,5 & 22,1 & 3,0 & 38,2 & 5,1 & 19,2 \\
\hline \multicolumn{7}{|l|}{ Local de ocorrência } \\
\hline Residência & 50,8 & 8,5 & 77,6 & 3,5 & 62,1 & 4,9 \\
\hline Habitação coletiva & 6,4 & 83,1 & 0,4 & 71,9 & 3,8 & 82,0 \\
\hline Via pública & 8,5 & 20,6 & 6,3 & 28,1 & 7,6 & 15,8 \\
\hline Comércio/Serviços/Indústria/Construção & 26,0 & 12,4 & 9,7 & 23,5 & 19,1 & 10,7 \\
\hline Outros & 6,3 & 20,9 & 5,2 & 28,6 & 5,8 & 16,9 \\
\hline Sem informação & 2,0 & - & 0,9 & - & 1,6 & - \\
\hline \multicolumn{7}{|l|}{ Natureza da lesão corporal } \\
\hline Queimadura & 94,0 & 1,4 & 96,2 & 1,5 & 95,0 & 1,1 \\
\hline Outros & 5,7 & 23,4 & 3,7 & 38,7 & 4,9 & 21,2 \\
\hline Sem informação & 0,2 & - & 0,1 & - & 0,2 & - \\
\hline \multicolumn{7}{|l|}{ Parte do corpo atingida } \\
\hline Cabeça e pescoço & 26,4 & 12,2 & 16,0 & 16,5 & 22,0 & 9,7 \\
\hline Membros superiores & 26,6 & 10,7 & 34,4 & 9,2 & 29,9 & 7,4 \\
\hline Membros inferiores & 13,9 & 14,4 & 24,1 & 12,3 & 18,2 & 10,2 \\
\hline Tronco & 11,8 & 18,0 & 12,8 & 21,2 & 12,2 & 16,7 \\
\hline Múltiplos órgãos/regiões & 19,8 & 25,0 & 12,0 & 16,8 & 16,5 & 18,7 \\
\hline Não se aplica *** & 1,5 & 46,7 & 0,7 & 81,9 & 1,2 & 40,4 \\
\hline Sem informação & - & - & - & - & - & - \\
\hline \multicolumn{7}{|l|}{ Evolução } \\
\hline Alta & 60,5 & 5,6 & 64,9 & 6,3 & 62,3 & 4,6 \\
\hline Encaminhamento ambulatorial & 11,5 & 21,4 & 14,6 & 28,1 & 12,8 & 22,3 \\
\hline Internação hospitalar & 21,7 & 17,3 & 13,1 & 18,3 & 18,1 & 14,3 \\
\hline Encaminhamento para outro serviço & 4,0 & 28,5 & 3,4 & 40,8 & 3,8 & 26,1 \\
\hline Evasão/Fuga & 1,0 & 51,8 & 2,0 & 45,5 & 1,4 & 34,4 \\
\hline Óbito & - & - & - & - & - & - \\
\hline Sem informação & 1,3 & - & 2,0 & - & 1,6 & - \\
\hline \multicolumn{7}{|l|}{ Agente causador } \\
\hline Fogo/Chama & 33,3 & 13,8 & 11,8 & 16,6 & 24,2 & 12,7 \\
\hline Substância quente & 30,7 & 10,8 & 61,3 & 5,4 & 43,6 & 6,4 \\
\hline Objeto quente & 9,9 & 16,8 & 9,2 & 19,6 & 9,6 & 13,5 \\
\hline Choque elétrico & 4,6 & 24,6 & 3,1 & 35,0 & 4,0 & 21,9 \\
\hline Substância química & 11,1 & 16,5 & 7,9 & 24,3 & 9,7 & 13,3 \\
\hline Outros & 7,7 & - & 4,0 & - & 6,1 & - \\
\hline Sem informação & 2,8 & - & 2,7 & - & 2,7 & - \\
\hline
\end{tabular}

(continua) 


\begin{tabular}{|c|c|c|c|c|c|c|}
\hline \multirow[t]{2}{*}{ Características } & \multicolumn{2}{|c|}{ Masculino $(n=446)$} & \multicolumn{2}{|c|}{ Feminino $(n=315)$} & \multicolumn{2}{|c|}{ Total $(N=761)$} \\
\hline & $\%$ & CV (\%) & $\%$ & CV (\%) & $\%$ & CV (\%) \\
\hline \multicolumn{7}{|c|}{ Período de atendimento } \\
\hline Manhã & 30,0 & 10,9 & 22,9 & 11,0 & 27,0 & 8,4 \\
\hline Tarde & 32,2 & 9,2 & 42,1 & 8,3 & 36,4 & 7,0 \\
\hline Noite & 29,4 & 11,3 & 32,8 & 11,0 & 30,8 & 9,1 \\
\hline Madrugada & 8,4 & 19,4 & 2,3 & 38,5 & 5,8 & 18,6 \\
\hline Sem informação & - & - & - & - & - & - \\
\hline \multicolumn{7}{|l|}{ Dia de atendimento } \\
\hline Domingo & 15,9 & 19,3 & 16,4 & 21,7 & 16,1 & 17,4 \\
\hline Segunda & 10,9 & 20,1 & 12,7 & 21,0 & 11,6 & 18,5 \\
\hline Terça & 8,2 & 23,3 & 10,9 & 22,5 & 9,3 & 19,3 \\
\hline Quarta & 12,3 & 24,6 & 12,9 & 29,9 & 12,6 & 24,6 \\
\hline Quinta & 14,8 & 27,0 & 14,0 & 25,4 & 14,4 & 25,1 \\
\hline Sexta & 22,7 & 23,2 & 17,4 & 19,1 & 20,4 & 18,5 \\
\hline Sábado & 14,6 & 22,1 & 14,0 & 21,9 & 14,3 & 19,9 \\
\hline Sem informação & 0,8 & - & 1,8 & - & 1,2 & - \\
\hline
\end{tabular}

* Serviço de Atendimento Móvel de Urgência (SAMU), ambulância, resgate;

** Uso de álcool informado pela vítima ou suspeito pelo entrevistador;

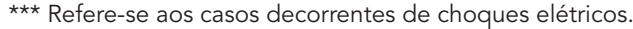

entorses, fraturas, entre outros. A maioria das vítimas recebeu alta $(62,3 \%)$ após o atendimento de emergência inicial, enquanto $18,1 \%$ foram encaminhadas para internação hospitalar e 16,6\% foram levadas para acompanhamento ambulatorial ou transferidas para outro serviço. Não houve a ocorrência de óbitos entre esses atendimentos. As maiores proporções de atendimento foram registradas nos turnos da tarde $(36,4 \%)$ e noite (30,8\%). Não foi observado padrão especial de ocorrência de casos em relação aos dias da semana, uma vez que as maiores proporções de atendimento ocorreram em sextas-feiras $(20,4 \%)$, domingos $(16,1 \%)$, quintas-feiras $(14,4 \%)$ e sábados $(14,3 \%)$.

As Figuras 1 e 2 mostram a distribuição das variáveis local de ocorrência e agente causador segundo a faixa etária, respectivamente, excluindo os registros sem informação. Observou-se que $96,5 \%$ das queimaduras em crianças de 0 a 4 anos ocorreram nas residências e que esta proporção vai diminuindo com o aumento da idade. Para os de 15 a 19 anos a residência como local de ocorrência respondeu por $46,3 \%$ dos casos e para $44,9 \%$ entre os que têm 20 e 29 anos de idade (Figura 1). Essa proporção voltou a crescer na faixa de 30 a 49 anos $(54,9 \%)$ e entre os de 50 anos e mais (66,9\%). O comércio, serviços, indústria e construção têm maior importância entre os adultos em idade produtiva uma vez que
$28,7 \%$ dos indivíduos com 20 a 29 anos e $25,6 \%$ daqueles com 30 a 49 anos sofreram queimaduras nestes locais.

Em relação ao agente causador (Figura 2), a substância quente foi responsável pela maior proporção de queimaduras em todas as faixas etárias, atingindo o valor mais alto para as crianças entre 0 e 4 anos (61\%) e mais baixo para aqueles com 15 a 19 anos (30,3\%). Queimaduras por fogo e/ou chamas ocuparam o segundo lugar em todas as faixas etárias; a proporção mais alta foi encontrada para os atendimentos nas idades de 30 a 49 anos $(31,1 \%)$ e mais baixa para as crianças entre 0 e 4 anos (13,2\%). Os maiores percentuais de queimaduras causadas por substâncias químicas foram encontrados nas faixas etárias que correspondem à idade produtiva, dos 10 aos 49 anos.

\section{Análise de correspondência}

A Tabela 3 apresenta as características demográficas e as características relacionadas ao evento que compõem cada dimensão. A "dimensão" deve ser entendida como um conjunto de categorias de variáveis que se mostraram associadas e que a "contribuição" mede a importância da categoria para explicar a associação verificada na composição da dimensão, sendo que quanto maior sua contribuição maior sua importância. 
Figura 1

Atendimentos decorrentes de queimaduras segundo faixa etária e local de ocorrência. Serviços de urgência e emergência selecionados em 23 capitais e no Distrito Federal, Brasil, setembro a novembro de 2009.

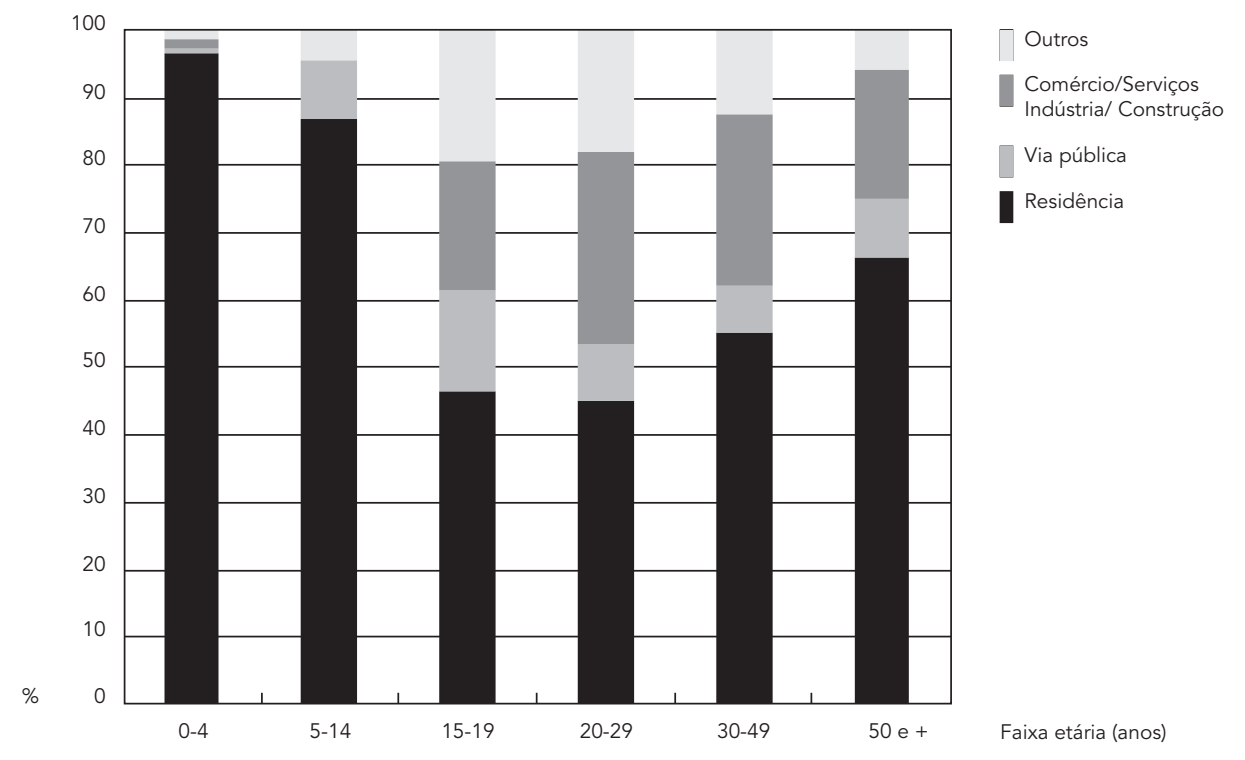

Figura 2

Atendimentos decorrentes de queimaduras segundo faixa etária e agente causador. Serviços de urgência e emergência selecionados em 23 capitais e no Distrito Federal, Brasil, setembro a novembro de 2009.

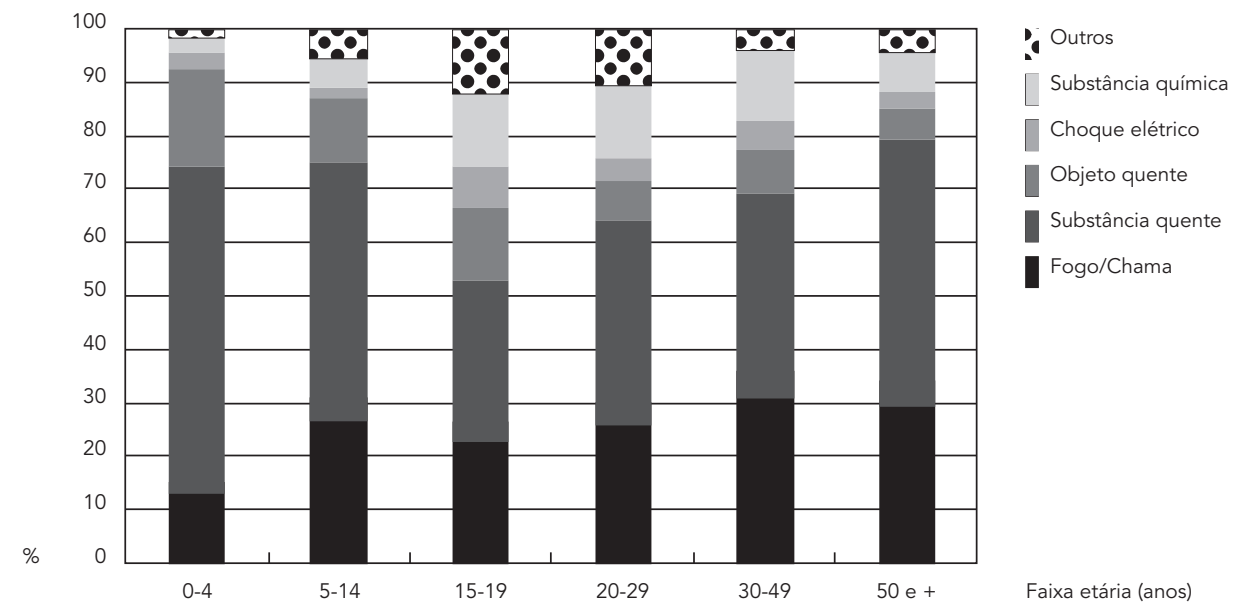


Coordenadas e contribuições das características demográficas das vítimas de queimaduras. Conjunto das 23 capitais e do Distrito Federal, Brasil, setembro a novembro de 2009.

\begin{tabular}{|c|c|c|c|c|c|c|c|c|c|c|}
\hline \multirow[t]{3}{*}{ Variáveis } & & \multirow[t]{3}{*}{$\begin{array}{l}\text { Frequência } \\
\text { relativa (A) }\end{array}$} & \multicolumn{2}{|c|}{$\begin{array}{l}\text { Coordenadas do } \\
\text { gráfico bi-plot }\end{array}$} & \multirow{3}{*}{$\begin{array}{c}\text { Proporção } \\
\text { da } \\
\text { variância } \\
\text { explicada } \\
\text { (D) }\end{array}$} & \multicolumn{5}{|c|}{ Contribuição } \\
\hline & & & \multirow[t]{2}{*}{1 (B) } & \multirow[t]{2}{*}{$2(C)$} & & \multicolumn{2}{|c|}{ do ponto } & \multicolumn{3}{|c|}{ da dimensão } \\
\hline & & & & & & $1(E)$ & $2(F)$ & 1 (G) & $2(H)$ & Total (I) \\
\hline Linha & Categorias & & & & & & & & & \\
\hline \multirow[t]{6}{*}{ Idade (anos) } & $0-4$ & 0,08 & 1,17 & $-0,12$ & 0,03 & 0,55 & 0,01 & 0,95 & 0,01 & 0,96 \\
\hline & $5-14$ & 0,06 & 0,68 & 0,28 & 0,01 & 0,13 & 0,05 & 0,69 & 0,06 & 0,75 \\
\hline & $15-19$ & 0,05 & $-0,48$ & 0,34 & 0,01 & 0,05 & 0,06 & 0,43 & 0,11 & 0,54 \\
\hline & $20-29$ & 0,12 & $-0,57$ & $-0,11$ & 0,01 & 0,18 & 0,02 & 0,77 & 0,01 & 0,79 \\
\hline & $30-49$ & 0,14 & $-0,30$ & 0,34 & 0,01 & 0,06 & 0,17 & 0,44 & 0,27 & 0,71 \\
\hline & $50 e+$ & 0,06 & $-0,06$ & $-0,82$ & 0,01 & 0,00 & 0,39 & 0,01 & 0,55 & 0,55 \\
\hline \multirow[t]{2}{*}{ Cor da pele } & Branca & 0,18 & $-0,14$ & $-0,34$ & 0,01 & 0,02 & 0,22 & 0,15 & 0,42 & 0,57 \\
\hline & Não branca & 0,31 & 0,07 & 0,17 & 0,00 & 0,01 & 0,09 & 0,15 & 0,42 & 0,57 \\
\hline Coluna & Categorias & & & & & & & & & \\
\hline \multirow[t]{6}{*}{ Tipo de queimadura } & Choque & 0,01 & $-0,29$ & 0,11 & 0,00 & 0,01 & 0,00 & 0,33 & 0,02 & 0,35 \\
\hline & Fogo & 0,08 & $-0,27$ & 0,25 & 0,00 & 0,03 & 0,05 & 0,25 & 0,10 & 0,35 \\
\hline & Substância quente & 0,16 & 0,25 & $-0,18$ & 0,00 & 0,05 & 0,05 & 0,77 & 0,18 & 0,96 \\
\hline & Substância química & 0,03 & $-0,74$ & 0,23 & 0,00 & 0,09 & 0,02 & 0,91 & 0,04 & 0,96 \\
\hline & Objeto quente & 0,03 & 0,62 & 0,47 & 0,00 & 0,06 & 0,08 & 0,61 & 0,17 & 0,77 \\
\hline & Outros & 0,02 & $-0,86$ & $-0,47$ & 0,01 & 0,08 & 0,05 & 0,39 & 0,06 & 0,45 \\
\hline Evolução na & Alta & 0,22 & $-0,18$ & 0,16 & 0,00 & 0,04 & 0,06 & 0,64 & 0,23 & 0,87 \\
\hline \multirow[t]{3}{*}{ emergência } & Ambulatório & 0,05 & $-0,10$ & $-1,12$ & 0,01 & 0,00 & 0,58 & 0,02 & 0,90 & 0,92 \\
\hline & Internação & 0,06 & 0,65 & 0,18 & 0,01 & 0,12 & 0,02 & 0,80 & 0,03 & 0,83 \\
\hline & Outro serviço & 0,01 & $-0,30$ & 0,07 & 0,00 & 0,01 & 0,00 & 0,14 & 0,00 & 0,15 \\
\hline \multirow[t]{4}{*}{ Local de ocorrência } & Residência & 0,22 & 0,44 & $-0,05$ & 0,01 & 0,21 & 0,01 & 0,98 & 0,01 & 0,99 \\
\hline & Comércio/Serviços & 0,05 & $-0,92$ & $-0,10$ & 0,01 & 0,20 & 0,01 & 0,94 & 0,01 & 0,95 \\
\hline & Indústria/Construção & 0,02 & $-0,95$ & $-0,15$ & 0,00 & 0,08 & 0,00 & 0,75 & 0,01 & 0,76 \\
\hline & Via pública & 0,03 & $-0,52$ & 0,57 & 0,00 & 0,03 & 0,09 & 0,47 & 0,27 & 0,74 \\
\hline
\end{tabular}

A dimensão 1 foi composta pelas categorias idade entre 0 e 4 anos, 20 a 29 anos (cujas contribuições correspondem a $55 \%$ e $18 \%$, respectivamente) e também pelas categorias internação, residência, comércio/serviço, e indústria/construção (cujas contribuições correspondem a $12 \%, 21 \%, 20 \%$ e $8 \%$, respectivamente). A dimensão 2 foi composta pelas categorias idade entre 30 e 49 anos e 50 anos ou mais, e raça/cor branca (cuja soma das contribuições corresponde a 78\%) e pelas categorias ambulatório (contribuição de 58\%).

A representação gráfica desse conjunto de variáveis foi possível devido à existência da associação entre as duas variáveis. A Figura 3 mostra por meio de linhas tracejadas três conjuntos de categorias que apresentaram associação. A primeira delas à esquerda mostra que os atendimentos nas faixas de 15 a 29 anos e de 30 a 49 estão associados com o fogo/chama e choque elétrico como agentes causadores, via pública como local de ocorrência e alta da emergência. Entre os adultos jovens na faixa de 20 e 29 anos essas queimaduras estiveram associadas com comércio/serviços e indústria/construção como local de ocorrência e outros como agente causador. $\mathrm{O}$ conjunto de categorias de variáveis na linha tracejada à direita mostra que as queimaduras em crianças de 0 a 4 anos e em crianças e adolescentes de 5 a 14 estiveram associadas com residência como local de ocorrência, substância quente e objeto quente como os agentes causadores da queimadura e internação hospitalar. O último conjunto de categorias tracejadas mostra a associação das queimaduras em indivíduos com 50 anos ou mais e o encaminhamento ambulatorial. Quanto à variável raça/cor, não foi 
Gráfico bi-plot das características demográficas e relacionadas às ocorrências das vítimas de queimaduras. Conjunto de 23 capitais e o Distrito Federal, Brasil, setembro a novembro de 2009

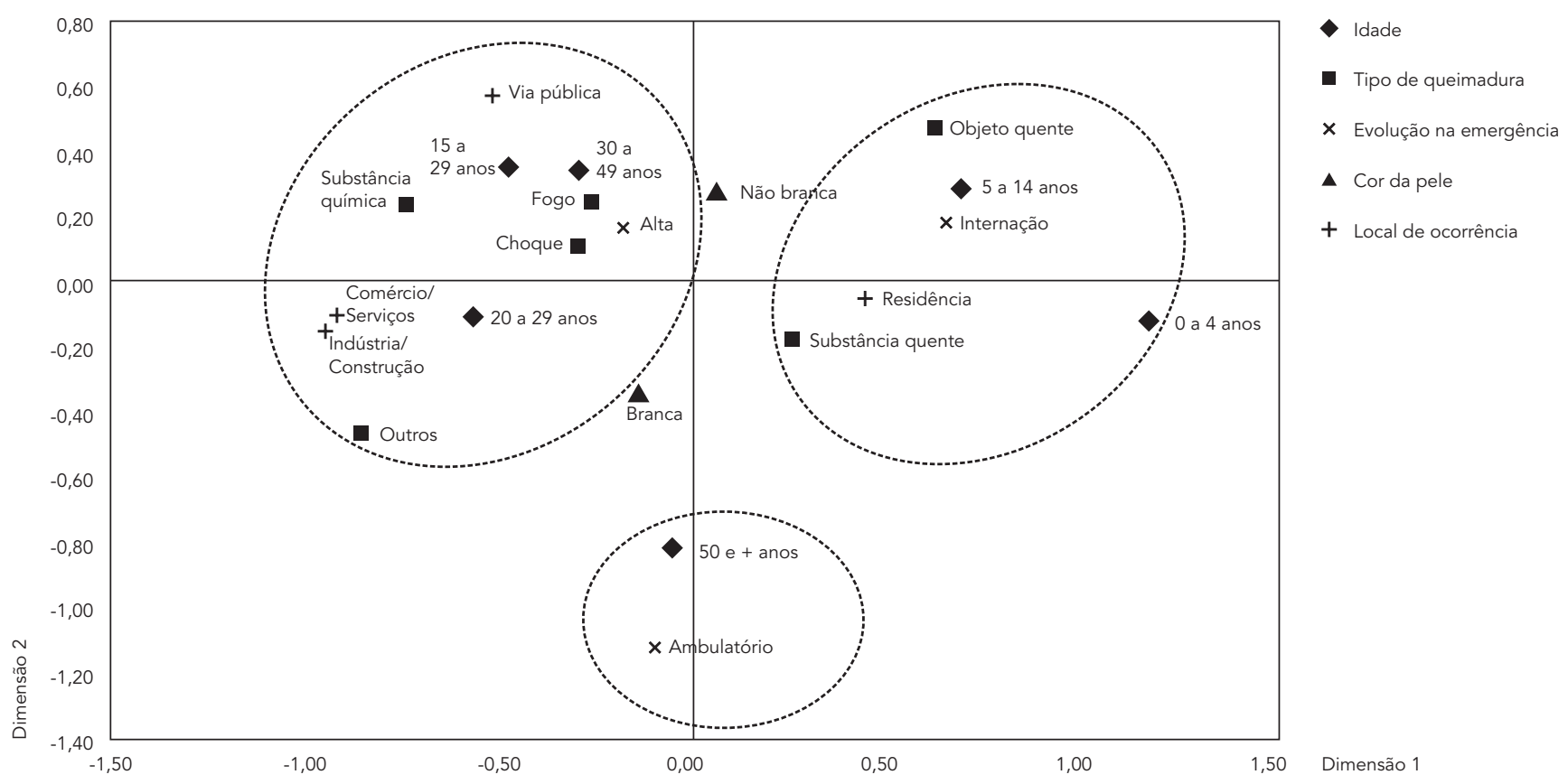

possível estabelecer adequadamente se as categorias branca e não branca estão associadas com outras variáveis, uma vez que suas posições no gráfico não permitiram conclusões.

\section{Discussão}

Os achados deste estudo que mostram que a metade dessas queimaduras ocorreu na residência da vítima são consistentes com os dados da literatura nacional 10,11 e internacional 12,13 , que apontam o ambiente doméstico como o local de ocorrência preferencial desses eventos. Certamente que as residências oferecem inúmeros riscos para essas ocorrências, dada a presença de uma gama de agentes inflamáveis (álcool, querosene, botijão de gás doméstico, lampiões, fósforos, isqueiros, velas), objetos quentes usados na atividade rotineira de preparar as refeições da família (fogão, panelas, churrasqueiras, alimentos) ou outros equipamentos domésticos (ferro de passar roupa, aquecedores), além de exposição à corrente elétrica (tomadas, instalações elétricas). Particularmente, as moradias precárias compostas por tábuas de madeira ou papelão apresentam maior vulnerabilidade para a exposição ao fogo e chamas. Considera-se necessário o desenvolvimento de estudos específicos para identificar melhor as circunstâncias em que essas queimaduras ocorrem em nosso meio para orientar, com bases epidemiológicas, os programas e as políticas de prevenção que devem ser implantados. Entre os fatores de risco apontados na literatura nacional foram encontrados o baixo nível socioeconômico e de instrução das mães e responsáveis pelas crianças, no momento do acidente, moradias pequenas para o número de residentes e equipamentos de cozinha precários 10 .

A grande proporção de queimaduras encontrada em crianças de 0 a 4 anos deve preocupar não somente pelo sofrimento e dor que representam, mas também pelas repercussões em termos de sequelas numa fase de rápido desenvolvimento físico, quando a perda de tecidos e/ ou sua contratura na evolução das lesões podem ocasionar danos permanentes mais graves. Os dados de internação hospitalar disponíveis no SIH/SUS para o ano de 2009 reforçam a importância das queimaduras nessa faixa etária, uma 
vez que na população em geral as queimaduras representaram 9\% do total de internações do grupo das causas externas, porém, entre as crianças de 0 a 4 anos elas representaram $17 \%$. Nessas internações, $84 \%$ foram decorrentes de exposição à corrente elétrica, contato com fonte de calor ou substância quente. Em relação ao agente causador, semelhante ao observado neste trabalho, em estudo conduzido em Londrina com pacientes internados ou atendidos em serviços de emergência também foi identificado que as escaldaduras (produzidas por substâncias quentes ou fontes de calor) causaram a maioria dessas queimaduras em menores de 15 anos, sendo que especificamente o líquido quente/fervente respondeu por quase a metade dos casos 14 . A importância das crianças entre as vítimas de queimaduras tendo como agente causador líquido quente também foi demonstrada em outros países 15 .

Entre as intervenções para redução desses eventos deve figurar aquelas voltadas para diminuir o acesso das crianças a qualquer agente causador de queimaduras presentes nas residências, por meio de um maior número de campanhas educativas voltadas para a população em geral e pais e crianças em particular. É preciso promover mudanças no ambiente doméstico que possibilitem prevenir esses eventos, como por exemplo, o uso de grades que impedem o acesso de crianças à cozinha. A supervisão inadequada das crianças é apontada na literatura internacional 12 como um fator de risco significante para mortes por causas externas em crianças. Essa questão deveria ser mais bem explorada em nosso meio, uma vez que a crescente participação das mulheres no mercado de trabalho 16 e como chefe de família pode significar que as mães que trabalham fora podem não contar com adultos para a supervisão de suas crianças. Desse modo, as políticas públicas que aumentam a disponibilidade e acesso a creches e escolas infantis podem contribuir para o controle e redução desses eventos.

Tanto o estudo descritivo quanto a análise de correspondência apontaram que as queimaduras nos indivíduos adultos jovens, em idade produtiva, na faixa de 20 a 29 anos, ocorreram em locais de trabalho classificados como comércio, serviços, indústria e construção. Embora a substância quente seja responsável por alta proporção de casos também nessa faixa, pode ser verificado que cresce de importância outros agentes como substância química, choque e fogo. Adicionalmente, os estudos acerca de queimaduras no ambiente de trabalho são escassos na literatura nacional, apontando ser esta também uma lacuna de conhecimento importante para pesquisas posteriores.
Outro aspecto que chamou atenção foi a alta proporção de internação hospitalar que chegou a $18,1 \%$ do total de vítimas de queimaduras. A análise realizada para outras causas externas registradas nesse mesmo banco mostrou que essa proporção é maior do que a verificada entre as vítimas de quedas $(7,5 \%)$ e até mesmo dos acidentes de trânsito (16,2\%). Os dados aqui apresentados não nos possibilitam explicar essa alta taxa e nem foi objeto do inquérito a avaliação da gravidade dessas lesões, porém, é sabido que para os casos que exigem internação, a assistência adequada e especializada ao paciente com queimadura em sua fase aguda tem um papel crucial na redução da mortalidade, das sequelas funcionais, estéticas e psicológicas. Certamente que as queimaduras profundas e extensas devem merecer maior cuidado pelo risco aumentado de infecção e sepse, devido ao acúmulo de tecidos necróticos que favorece a proliferação de agentes patogênicos 17,18,19. Do mesmo modo, a contratura dos tecidos atingidos, que ocorre durante a evolução das lesões e que pode levar a incapacidades físicas com limitação funcional, merece cuidados especializados. Por outro lado, os recursos e técnicas para o tratamento vêm evoluindo ao longo dos tempos, fazendo com que o estabelecimento de um número maior de centros especializados em cuidados aos pacientes que sofreram queimaduras deva figurar entre as estratégias necessárias a serem implantadas nessa área.

A interpretação dos dados do presente trabalho encontra-se sujeita a algumas limitações. A primeira delas é que os dados foram provenientes de uma amostra de conveniência e, portanto, não devem ser generalizados para o Brasil e nem possibilitam a construção de taxas. Outra limitação é que os dados apresentados referemse principalmente aos pacientes ambulatoriais ou que receberam alta, uma vez que os dados dos pacientes ambulatoriais e dos internados não foram analisados separadamente. O perfil epidemiológico dos pacientes internados pode ser diferente. Um estudo prospectivo conduzido em Brasília mostrou que entre os pacientes com complicações por infecções por fungos ou bactérias a mediana da superfície do corpo atingida foi de $14 \%$, o tempo médio de permanência destes casos foi de 12 dias com taxa de letalidade de $5 \%$ 19. Em outro trabalho conduzido em Uberaba, Minas Gerais, com pacientes cuja superfície corporal queimada foi maior (20,8\%), o tempo médio de internação chegou a 16,2 dias e a chama aberta foi o agente causador da maioria dos casos 20 . 


\section{Conclusões}

Os resultados aqui apresentados mostraram que do ponto de vista das características demográficas, a maioria das vítimas de queimaduras atendidas em serviços de urgência e emergência selecionados em todas as capitais do país pertencia ao sexo masculino, era composta por crianças menores de 10 anos ou adultos jovens na faixa de 20 a 29 anos, com escolaridade entre 0 e 4 anos ou 9 a 11 anos de estudos. Além disso, quase um terço desses atendimentos foi relacionado ao trabalho e quase a totalidade das lesões foi classificada como queimadura que atingiu especialmente os membros superiores e inferiores, cujo agente causador principal foi substância quente. A residência figurou como um importante local de ocorrência dessas queimaduras para todas as idades.

A análise de correspondência mostrou que os atendimentos nas faixas de 15 a 49 anos estiveram associados com o fogo/chama e choque elétrico como agentes causadores. Já as queimaduras entre os adultos jovens na faixa de 20 e 29 anos estiveram associadas com comércio/ serviços e indústria/construção como local de ocorrência. Na literatura nacional são escassos os estudos que abordam o problema das quei- maduras no ambiente de trabalho, configurando o problema como uma lacuna no conhecimento científico. Por isso, considera-se que devem ser desenvolvidas mais pesquisas para aumentar o conhecimento do problema, bem como a sua prevenção.

Adicionalmente, algumas lacunas de conhecimento foram identificadas, como por exemplo, a necessidade de conhecer melhor as circunstâncias em que essas queimaduras ocorreram e as queimaduras no ambiente de trabalho, o que deve ser objeto de estudos posteriores.

Considera-se que a abordagem do problema das queimaduras e o estabelecimento de atividades de prevenção e de tratamento especializado devem envolver uma ampla gama de profissionais com diferentes perfis, que atuam na área de saúde pública, clínica e saúde mental. Igualmente importante é promover o envolvimento da sociedade como um todo, especialmente os grupos sob maior risco, que são as crianças (por intermédio dos pais, responsáveis e educadores) e os trabalhadores. O setor legislativo, a mídia, entre outros, também desempenham um papel importante para que juntos consigamos chamar atenção para o problema e estabelecer as políticas públicas e medidas de prevenção mais adequadas ao nosso meio.

\section{Resumo}

O objetivo foi analisar os atendimentos por queimaduras em serviços de emergência, e fatores associados. Estudo transversal de 761 atendimentos coletados pelo Sistema de Vigilância de Violências e Acidentes em 2009. A maioria foi do sexo masculino (58,6\%); adultos de 30 a 49 anos $(23,1 \%)$ e crianças de 0 a 9 anos (23\%). A residencia foi o local de ocorrência mais frequente $(62,1 \%)$, especialmente para crianças e mulheres; em seguida comércio, serviços, indústria e construção (19,1\%), especialmente entre homens de 20 a 49 anos. Queimaduras no trabalho foram 29,1\% do total. Uso de álcool chegou a 5,1\%. Agentes causadores em todas as idades: substância quente (43,6\%) e fogo/chama (24,2\%); na faixa produtiva: substâncias químicas. As queimaduras entre 0 e 14 anos foram associadas com residência, substância e objeto quente e internação hospitalar; entre os de 15 a 49 anos associaram-se com fogo/chama e choque elétrico, via pública e alta da emergência. Estratégias de prevenção para crianças e trabalhadores devem ser implantadas.

Queimaduras; Serviços Médicos de Emergência; Causas Externas

\section{Colaboradores}

Os autores colaboraram igualmente na elaboração do artigo. 


\section{Referências}

1. Rossi LA, Camargo C, Santos CMNM, Barruffin RCP, Carvalho EC. A dor da queimadura: terrível para quem sente, estressante para quem cuida. Rev Latinoam Enferm 2000; 8:18-26.

2. Mock C, Peck M, Peden M, Krug E. A WHO plan for burn prevention and care. Geneva: World Health Organization; 2008.

3. Mascarenhas MDM, Silva MMA, Malta DC, Moura L, Macário EM, Gawryszewski VP, et al. Perfil epidemiológico dos atendimentos de emergência por violência no Sistema de Serviços Sentinelas de Vigilância de Violências e Acidentes (Viva) - Brasil, 2006. Epidemiol Serv Saúde 2009; 18:17-28.

4. Departamento de Análise de Situação de Saúde, Secretaria de Vigilância em Saúde, Ministério da Saúde. Viva: vigilância de violências e acidentes, 2009. Brasília: Ministério da Saúde; 2010.

5. Silva NN. Amostragem probabilística: um curso introdutório. 2a Ed. São Paulo: Edusp; 2001.

6. Mingoti SA. Análise de dados através de métodos estatísticos multivariados: uma abordagem aplicada. Belo Horizonte: Universidade Federal de Minas Gerais; 2005.

7. Souza AMR. Análise de correspondência [Dissertacão de Mestrado]. São Paulo: Instituto de Matemática e Estatística, Universidade de São Paulo; 1982.

8. Ramos EMLS, Almeida SS, Araújo AR. Segurança pública: uma abordagem estatística e computacional. Belém: EDUFPA; 2008.

9. Stata Corporation. Stata survey data reference manual. College Station: Stata Corporation; 2003.

10. Vendrusculo TM, Balieiro CRB, Echevarría-Guanilo ME, Farina Junior JA, Rossi LA. Burns in the domestic environment: characteristics and circumstances of accidents. Rev Latinoam Enferm 2010; 18:444-51.

11. Rossi LA, Ferreira E, Costa ECFB, Bergamasco EC, Camargo C. Prevenção de queimaduras: percepção de pacientes e de seus familiares. Rev Latinoam Enferm 2003; 11:36-42.
12. Warda LJ, Ballesteros MF. Interventions to prevent residential fire injury. In: Doll LS, Bonzo SE, Mercy JA, Sleet D, editors. Handbook of injury and violence prevention. New York: Springer; 2007. p. 97-115.

13. Haik J, Liran A, Tessone A, Givon A, Orenstein A, Peleg K. Burns in Israel: demographic, etiologic and clinical trends, 1997-2003. Isr Med Assoc J 2007; 9:659-62.

14. Martins CBG, Andrade SM. Queimaduras em crianças e adolescentes: análise da morbidade hospitalar e mortalidade. Acta Paul Enferm 2007; 20:464-9.

15. Brudvik C, Hoem EL, Luggenes B, Vindenes H. Burn injuries in children. Tidsskr Nor Laegeforen 2011; 131:20-3.

16. Instituto Brasileiro de Geografia e Estatística. Síntese de indicadores sociais: uma análise das condições de vida da população brasileira 2008. Rio de Janeiro: Instituto Brasileiro de Geografia e Estatística; 2008. (Estudos e Pesquisas, Informação Demográfica e Socioeconômica, 23).

17. Ferreira E, Lucas R, Rossi LA, Andrade D. Curativo do paciente queimado: uma revisão de literatura. Rev Esc Enferm USP 2003; 37:44-51.

18. Macedo JLS, Rosa SC, Macedo KCS, Castro C. Fatores de risco da sepse em pacientes queimados. Rev Col Bras Cir 2005; 32:173-7.

19. Soares de Macedo JL, Santos JB. Nosocomial infections in a Brazilian Burn Unit. Burns 2006; 32 477-81.

20. Montes SF, Barbosa MH, Sousa Neto AL. Aspectos clínicos e epidemiológicos de pacientes queimados internados em um Hospital de Ensino. Rev Esc Enferm USP 2011; 45:369-73.

Recebido em 04/Ago/2011

Versão final reapresentada em 29/Nov/2011

Aprovado em 13/Dez/2011 